\title{
Papel atual das estratégias ventilatórias protetoras no período perioperatorio: artigo de revisão
}

\section{Protective ventilatories in the perioperative period: review article}

Inara Nobre de Castro ${ }^{1}$. Lorena Antônia Sales de Vasconcelos Oliveira² ${ }^{2}$ Flavio Lobo Maia ${ }^{3}$. Juliana Rosa Melo ${ }^{1}$. Fernanda Paula Cavalcante ${ }^{4}$.

1 Médica residente do Programa de Anestesiologia, Universidade Federal do Ceará (UFC), Fortaleza, Ceará, Brasil. 2 Mestre em cirurgia pela Universidade Federal do Ceará (UFC), Preceptora do Programa de Anestesiologia e Médica do Hospital Universitário Walter Cantídio (HUWC), Fortaleza, Ceará, Brasil. 3 Título Superior em Anestesiologia pela Sociedade Brasileira de Anestesiologia, Preceptor do Programa de Anestesiologia e Médico do Hospital Universitário Walter Cantídio (HUWC). 4 Título Superior em Anestesiologia pela Sociedade Brasileira de Anestesiologia, Médica Anestesiologista responsável pelo Programa de Anestesiologia do Hospital Universitário Walter Cantídio (HUWC).

\section{RESUMO}

Os fármacos anestésicos utilizados para indução e manutenção de anestesia geral provocam alterações da dinâmica respiratória, fazendo-se necessário o uso de estratégias ventilatórias perioperatórias. A ventilação mecânica, apesar de ser uma terapia de suporte essencial, não é isenta de riscos. Dentre estes, podemos citar complicações pulmonares pós-operatórias (CPPs), que apresentam alta prevalência e potenciais implicações graves. Fatores cirúrgicos, anestésicos e do paciente contribuem para o desenvolvimento de CPPs. Essa revisão faz uma análise de artigos publicados recentemente na literatura sobre ventilação mecânica e suas consequências na morbimortalidade em pacientes cirúrgicos. Por várias décadas e até recentemente, o manejo ventilatório durante cirurgia esteve associado a altos volumes corrente (VC), ausência de pressão expiratória positiva (PEEP) e altas frações inspiradas de oxigênio $\left(\mathrm{FiO}_{2}\right)$. Avanços crescentes na compreensão da fisiopatologia de lesão pulmonar induzida por ventilação mecânica, por meio de estudos experimentais, observacionais e randomizados, indicam a necessidade de se instituir estratégias ventilatórias protetoras perioperatórias. Estas estratégias incluem, de maneira geral, a utilização de baixos VC, uso de PEEP, manobras de recrutamento alveolar e baixa $\mathrm{FiO}_{2}$.

Palavras-chave: Anestesia. Ventilação. Período intraoperatório. Complicações pós-operatórias.

\section{ABSTRACT}

The use of anesthetic drugs for induction and maintenance of general anesthesia cause changes in respiratory dynamics, making it necessary to use perioperative ventilatory strategies. Mechanical ventilation, although it is an essential supportive therapy, is not without risk. Among these, they can mention postoperative pulmonary complications (PPCs), which have high prevalence and potential for serious implications. Many factors such as surgical, anesthetic and patient ones contribute to the development of PPCs. This review analyzes updated published literature on mechanical ventilation and its consequences on morbidity and mortality in surgical patients. For several decades and until recently, ventilatory management during surgery was linked with high tidal volumes (TV), absence of positive expiratory pressure (PEEP) and high inspired oxygen fractions $\left(\mathrm{FiO}_{2}\right)$. Increasing advances in the understanding of physiopathology of mechanical ventilation-induced lung injury through experimental, observational, and randomized studies indicate a need to institute perioperative protective ventilation. These strategies generally include the use of low $\mathrm{CV}$, use of PEEP, alveolar recruitment maneuvers and low $\mathrm{FiO}_{2}$.

Keywords: Anesthesia. Ventilation. Intraoperative period. Postoperative complications.

Autor correspondente: Inara Nobre de Castro, Rua Guilherme Rocha, 1299, apartamento 801, Centro, Fortaleza, Ceará. CEP: $60030-141$. Telefone: +55 88 996109683. E-mail: inara.nobre@yahoo.com.br

Conflito de interesses: Não há qualquer conflito de interesses por parte de qualquer um dos autores.

Recebido em: 12 Abr 2017; Revisado em: 27 Jun 2017; Aceito em: 28 Jun 2017. 


\section{INTRODUÇÃO}

A ventilação mecânica (VM) é uma terapia de suporte essencial para manter uma oxigenação adequada durante a anestesia geral. Apesar de ser uma intervenção necessária, cada vez mais são reconhecidos os potenciais efeitos nocivos associados à VM. ${ }^{1}$ Evidencias clínicas indicaram que a VM pode resultar em lesão difusa de células alveolares, edema pulmonar, recrutamento celular de células inflamatórias e produção de citocinas, culminando com complicações pulmonares e extrapulmonares. As complicações pulmonares pósoperatórias (CPPs) constituem a principal causa de morbidade perioperatória e mortalidade em pacientes após anestesia geral. ${ }^{2}$

Nas últimas décadas, estudos sobre VM em pacientes com síndrome do desconforto respiratório agudo (SDRA) levaram ao desenvolvimento de uma série de 'estratégias ventilatórias protetoras' (EVP), capazes de diminuir a mortalidade em pacientes em unidades de terapia intensiva (UTI). ${ }^{3,4}$ Essas estratégias já foram bastante estudadas em pacientes críticos, porém, sua relevância no perioperatório está sendo mais recentemente considerada, sendo aplicada inclusive em pacientes sem comorbidades, como em pacientes cirúrgicos submetidos a anestesia geral.

Atualmente, uma abordagem moderna para a gestão da ventilação leva em consideração principalmente a redução do volume corrente, somado também ajustes em outros parâmetros como aplicação de pressão positiva expiratória final (PEEP), manobras de recrutamento alveolar e menor fração inspirada de oxigênio $\left(\mathrm{FiO}_{2}\right){ }^{5}$

Embora estudos mostrem que altos volumes correntes (VC) sejam deletérios aos pulmões, acreditava-se que sua utilização no intraoperatório por curto período (horas) era segura. Porém, vários ensaios clínicos de ventilação intraoperatória sugeriram que uma redução do $\mathrm{VC}$ poderia melhorar a mecânica respiratória e oxigenação. Além disso, recentes metanálises ${ }^{2,5,6}$ e ensaios controlados randomizados $(\mathrm{ECR})^{7,8,9}$ sugerem que o uso de EVP durante o intraoperatório reduz a incidência de complicações pulmonares.

Deste modo, a aplicação de EVP deve ser indicada a todos os pacientes cirúrgicos submetidos a anestesia geral, resultando em diminuição de CPPs e consequente diminuição da morbidade perioperatoria, redução do tempo de internação e custos hospitalares. ${ }^{10}$

Diante da relevância do tema, este artigo fornece uma revisão da literatura sobre os benefícios da ventilação mecânica protetora intraoperatória, na redução do desenvolvimento de complicações pulmonares pós-operatórias.

\section{METODOLOGIA}

Trata-se de um artigo de revisão sobre os benefícios da ventilação mecânica protetora intraoperatória, na redução do desenvolvimento de complicações pulmonares pós-operatórias e consequente redução da morbimortalidade perioperatória. Foi realizada pesquisa de literatura online no database da MEDLINE
(PUBMED), selecionados artigos publicados nos últimos cinco anos, com as palavras-chave: 1 . ventilação mecânica, 2 . perioperatório e 3. complicações pulmonares. Incluiu-se artigos originais, artigos de revisão, revisão sistemática e diretrizes escritos na língua inglesa, resultando em 25 artigos.

\section{DISCUSSÃO}

\section{Efeitos da anestesia no sistema respiratório}

A mecânica respiratória sofre influência de diversos fatores, entre eles, podemos citar: o peso, a idade, a posição do corpo (vertical versus supina) e o uso de fármacos. A maioria das medicações anestésicas gera perda do tônus muscular, levando a um deslocamento cranial do principal músculo da respiração, o diafragma, e consequente diminuição dos volumes pulmonares. Entre estes, a redução da capacidade residual funcional (CRF), que promove o fechamento de vias aéreas e formação de atelectasia ${ }^{11}$ (Gráfico 1).

Gráfico 1. Efeito da idade, decúbito dorsal e anestesia na capacidade residual funcional(CRF) e capacidade de fechamento(CR).

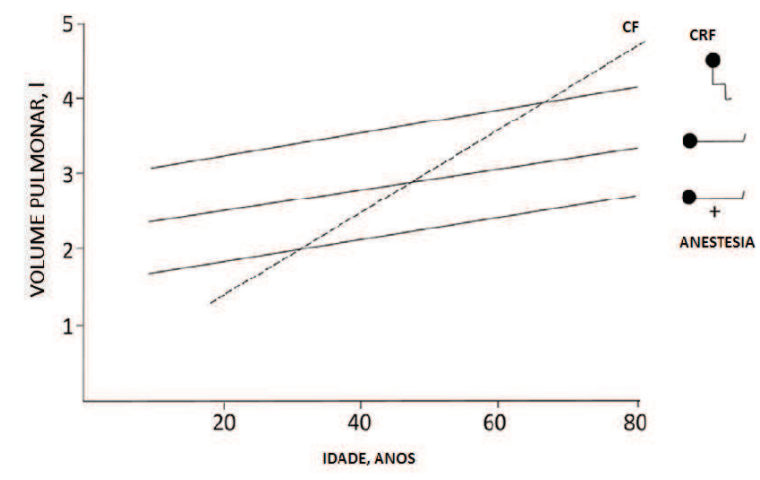

Gráfico adaptado de: Hedenstierna G, Edmark L, Effects of anesthesia on the respiratory system. Best Pract Res Clin Anaesthesiol. 2015;29(3):273-84.

Atelectasias podem ocorrer em cerca de $90 \%$ dos pacientes anestesiados, principalmente em bases pulmonares, tanto nos pacientes mantidos em respiração espontânea, como sob ventilação mecânica. Cerca de 10 a $20 \%$ da área pulmonar total entra em colapso durante o ato anestésico. ${ }^{11,12}$ Mesmo várias horas após cirurgia cardíaca e torácica, mais da metade do pulmão pode ainda estar colapsado. A área de pulmão atelectasiado, pode se tornar sítio propenso a infecção e contribuir para o surgimento de complicações pulmonares pós-operatórias (CPPs). ${ }^{11,13,14}$

\section{Complicações pulmonares pós-operatórias e fatores de risco}

Existem diversas definições de CPPs na literatura. Com o intuito de padronizar os critérios diagnósticos de CPPs, uma força-tarefa da Sociedade Europeia de Medicina Intensiva e Sociedade Europeia de Anestesiologia, propôs os critérios contidos no Quadro 1. ${ }^{14}$ Fatores de risco para CPPs podem ser identificados em uma avaliação pré-operatória, com o 
objetivo de redução do risco de desenvolvimento de CPPs perioperatórias. ${ }^{14,15}$ Quanto mais cedo forem identificados esses fatores de risco, maior a probabilidade de serem

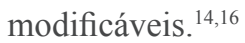

Quadro 1. Definição de complicações pulmonares pós-operatórias.

\begin{tabular}{|c|c|}
\hline Complicação & Definição \\
\hline Infecção respiratória & $\begin{array}{l}\text { Pacientes que receberam } \\
\text { antibióticos por suspeita de } \\
\text { infecção respiratória } \\
\text { Presença de um ou mais dos } \\
\text { seguintes: } \\
\text { - Escarro novo ou modificado } \\
\text { - Opacidade pulmonar nova ou } \\
\text { modificada } \\
\text { - Febre } \\
\text { - Leucócitos }>12 \mathrm{mil}\end{array}$ \\
\hline Falência Respiratória & $\begin{array}{l}\text { - } \mathrm{PaO}_{2} \text { pós-operatória }<60 \mathrm{mmHg} \\
\mathrm{em} \mathrm{ar} \text { ambiente; ou } \\
\text { - } \quad \mathrm{SpO}_{2}<90 \% \text { e necessitando de } \\
\text { oxigenoterapia }\end{array}$ \\
\hline Derrame pleural & $\begin{array}{l}\text { Radiografia de pulmão demostrando: } \\
\text { - Apagamento de seio } \\
\text { costofrênico } \\
\text { Perda da nitidez da silhueta do } \\
\text { hemidiafragma ipsilateral na } \\
\text { posição superior direita } \\
\text { - Evidência de deslocamento } \\
\text { de estruturas anatômicas } \\
\text { adjacentes } \\
\text { (na posição supina) opacidade } \\
\text { nebulosa em um hemitórax com } \\
\text { sombras vasculares preservadas }\end{array}$ \\
\hline Atelectasias & $\begin{array}{l}\text { - Opacificação pulmonar com } \\
\text { deslocamento do mediastino, } \\
\text { hilo ou hemidiafragma em } \\
\text { direção à área afetada, e } \\
\text { - Superinflação compensatória } \\
\text { no pulmão não-atelectasiado } \\
\text { adjacente }\end{array}$ \\
\hline Pneumotórax & $\begin{array}{l}\text { Ar no espaço pleural sem trama vascular } \\
\text { em torno da pleura visceral }\end{array}$ \\
\hline Broncoespasmo & $\begin{array}{l}\text { Detecção recente de sibilos à respiração } \\
\text { expiratória tratado com broncodilatadores }\end{array}$ \\
\hline Pneumonite aspirativa & $\begin{array}{l}\text { Injúria pulmonar aguda após inalação de } \\
\text { conteúdo gástrico regurgitado }\end{array}$ \\
\hline
\end{tabular}

Quadro adaptado de: Gallart L, Canet J. Post-operative pulmonary complications: understanding definitions and risk assessment. Best Practice \& Research Clinical Anaesthesiology. 2015;29:315-330.

A idade avançada é um dos fatores de risco mais frequentemente descritos para CPPs. ${ }^{12,14-16}$ A fragilidade (maior vulnerabilidade aos agentes estressores) é um estado que compromete a capacidade do indivíduo para se recuperar após uma cirurgia. ${ }^{14}$ Idade acima de 65 anos dobrou o risco de CPPs em alguns estudos, como um preditor independente, baseado em alterações pulmonares relacionadas a idade..$^{12,13}$

Outro fator de risco para CPPS avaliado é a classificação da American Society of Anesthesiologists (ASA), que é amplamente conhecida e pode ser definida rapidamente. Classificação ASA estima, não somente o risco de CPPs, como também o risco global para o desenvolvimento de outras complicações. ${ }^{12,14-16} \mathrm{O}$ risco de CPPs aumenta em até três vezes para os pacientes com doença sistêmica moderada ou grave (ASA classe III) e em até cinco vezes em pacientes moribundos (ASA classe IV). ${ }^{13}$

O tabagismo também é fator de risco para o desenvolvimento de CCPs. ${ }^{12-16}$ Fumantes ativos têm um aumento nas secreções traqueobronquiais e uma diminuição da atividade ciliar. Eles são dependentes de tosse para a remoção de secreções, e podem precisar de maior tempo de desmame da ventilação mecânica na unidade de terapia intensiva (UTI). ${ }^{12,13,16}$

O American College of Physicians (ACP) considera a doença pulmonar obstrutiva crônica (DPOC) como fator de risco mais comumente relacionado a CPPs. ${ }^{12,14-16}$ Quando se considera a presença de sintomas da DPOC (tosse, expectoração, dispneia e sibilos), esses se correlacionam mais fielmente com o risco de CPPs do que quando a análise dessa variável é realizada de forma dicotômica (tem ou não tem a doença). ${ }^{14}$ Devido a sua relevância, DPOC será abordado mais à frente nesse trabalho.

O teste de tosse é um teste clínico de fácil realização que requer que o paciente respire fundo e tussa uma vez. É considerado positivo se o paciente continua a tossir. ${ }^{12,14}$ Em um estudo prospectivo, o teste positivo e a baixa saturação periférica de oxigênio $\left(\mathrm{SpO}_{2}\right)$ foram associados a CPPs..$^{12,14,16}$

A infecção respiratória no último mês só foi incluída como variável no estudo prospectivo multicêntrico para o escore ARISCAT (The Assess Respiratory Risk in Surgical Patients in Catalonia Group $)^{12,14}$ e no estudo PERISCOPE (Prospective Evaluation of a Risk Score for postoperative pulmonary Complications in Europe). ${ }^{17}$ Sugere-se que uma infecção respiratória recente justificaria adiar uma cirurgia eletiva, principalmente se outros fatores de risco também estão presentes. ${ }^{14,16,17}$

Insuficiência cardíaca congestiva (ICC) é um forte preditor de CPPs, com odds ratio (OR) para mortalidade de cerca de 2, e quanto maior a gravidade da ICC, maior o risco. ${ }^{14,15}$

Outras variáveis como desnutrição, obesidade, queda dos níveis de albumina sérica, apneia obstrutiva do sono (AOS), anemia, transfusão, sepse, consumo de álcool, insuficiência renal e doença hepática crônica são fatores de risco de CPPs. ${ }^{12,14-17}$ Diabetes mellitus, uso de corticosteroide, câncer, doença do refluxo gastresofágico (DRGE), sexo masculino e hipertensão arterial não são apoiados com boa evidencia nas diretrizes do ACP de 2006, necessitam de estudos futuros para identificar se aumentam ou não o risco de desenvolvimento de CPPs. ${ }^{14}$

Fatores relacionados ao procedimento cirúrgico também podem aumentar o risco de CPPs. Cirurgia cardíaca, torácica e abdominal alta são preditores positivos fortes para o desenvolvimento de CPPs. ${ }^{14-16}$ Tal fato se dá provavelmente 
devido à proximidade cirúrgica com diafragma e outros músculos respiratórios, o que pode ocasionar dor a respiração profunda e consequente atelectasias. ${ }^{14,16}$ Ainda de acordo com as diretrizes da $\mathrm{ACP}$, cirurgia vascular, neurocirurgia, cirurgia da cabeça e pescoço, cirurgia de emergência e cirurgias prolongadas oferecem bom nível de evidência para CPPs. ${ }^{12,14,15}$

Quanto a técnica anestésica, a anestesia geral foi associada a risco de CPPs, ${ }^{11,17}$ porém ensaios clínicos randomizados posteriores não demostraram tal relação. ${ }^{14}$ Provavelmente um melhor manejo anestésico com novos fármacos, ventiladores mecânicos modernos e melhor monitorização teria diminuído o risco hipotético relacionado à anestesia geral. ${ }^{14}$ No entanto, alguns estudos demonstram que evitar anestesia geral em pacientes com DPOC e obesidade tem benefícios na redução de CPPs. ${ }^{12,13}$

\section{Mecanismos de lesão pulmonar induzida por ventilador}

Lesão pulmonar induzida pelo ventilador (LPIV) é o resultado de uma interação complexa entre a força mecânica que atua sobre as estruturas do pulmão, tais como células epiteliais tipo I e II, células endoteliais, macrófagos e a matriz extracelular, durante a ventilação mecânica $(\mathrm{VM}) .{ }^{18} \mathrm{~A}$ superdistensão regional dos alvéolos causada pela aplicação de grandes volumes ou pressões pode provocar abrasão do epitélio, inativação de surfactante e deformação de células e matriz extracelular. ${ }^{16,18}$

Essa deformação cíclica alveolar leva a lesões que geram perturbações proporcionais à frequência, amplitude e duração do estresse tecidual. Estudo demonstrou que a maioria das lesões é iniciada ainda nos primeiros minutos da VM. ${ }^{18}$ Nesse contexto, o estresse e a tensão sobre o tecido saudável ou lesado induz a liberação de mediadores inflamatórios (interleucinas, citocinas, espécies reativas de oxigênio, entre outras) que iniciam uma cascata de eventos que culmina com lesão pulmonar. ${ }^{3,16,18}$ Somando-se a isso, a sobrecarga hídrica, relacionada a reposição volêmica excessiva, desorganiza o interstício, contribuindo ainda mais para formação de edema pulmonar severo e LPIV. ${ }^{1,12,16}$

\section{Estratégias ventilatórias protetoras}

Por muitos anos, a ventilação mecânica com altos volumes correntes $(10-15 \mathrm{ml} / \mathrm{kg})$ era uma prática comum em salas cirúrgicas, ${ }^{5}$ pois seguiam a tradição que um alto $\mathrm{VC}$ durante a cirurgia preveniria a formação de atelectasias, ${ }^{5,19}$ acidose respiratória e redução da dessaturação intraoperatória. ${ }^{5}$ Este conceito foi baseado em um famoso estudo em 1963 realizado por Bendixen at al. ${ }^{20}$ que influenciou o manejo ventilatório por décadas. Esse trabalho ainda é citado em livros de referência atuais, mas agora é questionado. ${ }^{5,21}$

Avanços crescentes na compreensão dos mecanismos da LPIV por meio de estudos em pacientes com síndrome do desconforto respiratório agudo (SDRA) levaram ao desenvolvimento de uma série de 'estratégias ventilatórias protetoras' (EVP), capazes de diminuir a morbimortalidade em doentes críticos. ${ }^{5,19,21}$ Essas EVP foram mais recentemente aplicadas também para ventilação em pulmões saudáveis como bom nível de evidência para redução de CPPs. ${ }^{5,18,19,21}$ A abordagem moderna para a gestão da ventilação leva em consideração não apenas a redução do VC, mas também ajustes em outros parâmetros como manobras de recrutamento alveolar, menor $\mathrm{FiO}_{2}$ e a aplicação de PEEP., $5,12,191$

Embora o uso de manobra de recrutamento (MR) seja descrito há anos, ainda não está claro quando e como essas manobras devem ser realizadas durante a cirurgia e se há uma pressão ideal nas vias aéreas para causar o recrutamento. A maioria dos médicos realizam a técnica manual de "apertar o balão" com pressão mantida a 30 ou $40 \mathrm{~cm}$ de $\mathrm{H}_{2} \mathrm{O}$ por cerca de 15 a 30 segundos. ${ }^{12,19,21,22}$ Outra técnica envolve o aumento gradual da PEEP de 5 em $5 \mathrm{~cm}$ de $\mathrm{H}_{2} \mathrm{O}$ até que um platô de $30 \mathrm{a} 40 \mathrm{~cm}$ de $\mathrm{H} 2 \mathrm{O}$ seja alcançado, em seguida se desmama a PEEP lentamente. ${ }^{19}$ Deve-se levar em consideração que em pacientes obesos mórbidos, a pressão de recrutamento convencional de 30 e $40 \mathrm{~cm}$ de $\mathrm{H} 2 \mathrm{O}$ pode ser inadequada para o recrutamento total dos pulmões: pressões de até $60 \mathrm{~cm}$ de $\mathrm{H} 2 \mathrm{O}$ podem ser necessárias. ${ }^{19}$

Quanto a $\mathrm{FiO}_{2}$, sabe-se que a hiperoxia é danosa aos pulmões, podendo ocasionar atelectasia de reabsorção. Durante uma intubação orotraqueal planejada ou emergencial existe um risco não insignificante de hipóxia. ${ }^{11,19}$ Certos subgrupos são mais propensos a sofrerem esse evento, como obesos, ${ }^{11,12,16,19}$ gestantes $^{11}$ e crianças $^{22}$ por diminuição da CRF. Além disso, portadores de doença cerebrovascular, epilepsia e doença coronariana são menos tolerantes a uma hipoxemia sistêmica, podendo ter consequências trágicas na sua ocorrência. ${ }^{11}$

Sabe-se que uma pré-oxigenação com alta $\mathrm{FiO}_{2}(0,8$ a 1$)$ pode induzir atelectasia por desnitrogenação, todavia um recente estudo randomizado mostrou que isso não influencia na CRF no pós-operatório. Portanto, os benefícios de se atingir uma boa oxigenação no momento da indução, prolongando o tempo de janela para uma intubação segura, superam esse risco. ${ }^{10}$ No entanto, após a indução aconselha-se o uso de $\mathrm{FiO}_{2}$ ente 0,3 a 0,4 , pois o risco de hipoxemia sistêmica é menor após a intubação orotraqueal. ${ }^{12}$

Quanto a PEEP, vários estudos experimentais demonstraram que a ventilação com PEEP (com ou sem manobras de recrutamento) melhora a aeração pulmonar e a oxigenação. ${ }^{5,12,18,22}$ Contudo, a PEEP também pode levar à distensão excessiva de regiões pulmonares não dependentes e comprometimento hemodinâmico. ${ }^{5,19,13}$ Um valor ideal para a PEEP ainda é tema de diversos estudos. . $2,4,5,19,21-23^{-1}$

Uma série de ensaios controlados randomizados confirmaram que a ventilação intraoperatória com VCs elevados aumenta a ocorrência de CPPs. ${ }^{5,13}$ Futier et al. relataram os benefícios de baixo VC com PEEP e MR, o que reduziu complicações pulmonares e encurtou o tempo de permanência hospitalar em comparação com o grupo controle. ${ }^{5}$ No entanto, o estudo PROVHILO $^{24}$ comparou dois grupos de paciente com $\mathrm{VC}$ baixo $(8 \mathrm{ml} / \mathrm{kg})$, um grupo com níveis de PEEP entre $12 \mathrm{cmH}_{2} \mathrm{O}$ e outro com PEEP de $2 \mathrm{cmH}_{2} \mathrm{O}$. O estudo mostrou 
que a incidência de complicações pulmonares pós-operatórias nos primeiros 5 dias após a cirurgia é comparável entre os dois grupos, com ambos os grupos submetidos a cirurgia abdominal aberta sob anestesia geral. Como VC foi o mesmo nos dois grupos, o resultado só poderia ser atribuído à PEEP aplicado durante a cirurgia. ${ }^{24}$

Mais recentemente, um estudo multicêntrico francês controlado randomizado, o estudo IMPROVE, demonstrou benefícios pulmonares e extrapulmonares significativos com a aplicação de EVP. Foi realizada comparação entre dois grupos: um com VC entre 6 e $8 \mathrm{~mL} / \mathrm{kg}$ do peso corporal previsto, PEEP entre 6 e $8 \mathrm{cmH} 2 \mathrm{O}$ e MR perioperatório a cada 30 minutos em comparação com outro grupo com VC entre 10 e $12 \mathrm{~mL} / \mathrm{kg}$ do peso corporal previsto, PEEP de $0 \mathrm{cmH} 2 \mathrm{O}$ e nenhuma manobra de recrutamento. ${ }^{21} \mathrm{O}$ primeiro grupo apresentou menor incidência de CPPs. Outros trabalhos que demostram benefícios da ventilação mecânica protetora são ilustrados na Tabela 1 .

\section{Modos de ventilação}

Atualmente, com o avanço da tecnologia, os ventiladores modernos dispõem de vários modos de assistência ventilatória. ${ }^{19}$ O Quadro 2 descreve os principais modelos utilizados. ${ }^{19} \mathrm{O}$ impacto do modo de ventilação na incidência de CPPs entre ventilação pressão controlada (PCV) e ventilação volume controlada (VCV) foi analisado em estudos retrospectivos e em ensaios clínicos randomizados, no entanto os resultados são contraditórios. ${ }^{19}$ Não existe uma evidência forte até o momento que um modo seja superior ao outro na prevenção de complicações. O que parece realmente importante é o uso de EVP. ${ }^{19}$ Exceto ventilação em pacientes pediátricos ${ }^{22}$ e pacientes com DPOC, ${ }^{13}$ os quais parecem se beneficiar com o modo PCV.

Tabela 1. Forest plot. Comparação entre ventilação protetora versus convencional.

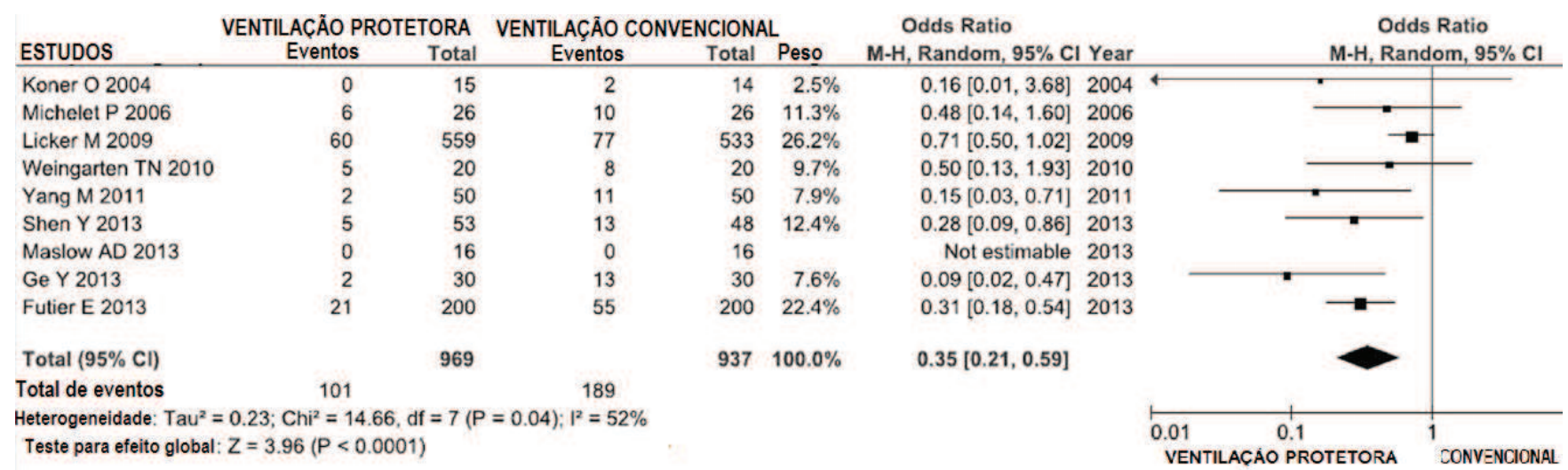

Tabela adaptada de: Serpa A Neto, Schultz MJ, Abreu MG. Intraoperative ventilation strategies to prevent postoperative pulmonary complications: systematic review, meta-analysis, and trial sequential analysis. Best Practice \& Research Clinical Anaesthesiology. 2015;29:331-340.

Quadro 02. Principais modos ventilatórios usados em centro cirúrgico.

\begin{tabular}{|c|c|c|c|}
\hline Modo & Tipo & Detalhes & Uso em anestesia \\
\hline $\begin{array}{l}\text { V-CMV (ventilação mandatória } \\
\text { volume contínuo), } \\
\text { VCV (ventilação volume controlado) }\end{array}$ & Mandatória & $\begin{array}{l}\text { Ventilação controlada, limitada a } \\
\text { fluxo, ciclagem a tempo }\end{array}$ & $\begin{array}{l}\text { Modo ventilatório mais comum, fornece } \\
\text { bom controle de VC, especialmente com } \\
\text { máquinas modernas de anestesia que } \\
\text { fornecem compensação }\end{array}$ \\
\hline $\begin{array}{l}\text { P-CMV (ventilação mandatória } \\
\text { pressão contínua), } \\
\text { PCV (ventilação controlada à } \\
\text { pressão) }\end{array}$ & Mandatória & $\begin{array}{l}\text { Ventilação controlada, limitada à } \\
\text { pressão de via aérea, ciclagem a } \\
\text { tempo }\end{array}$ & $\begin{array}{l}\text { Modo de ventilação comum, fornece bom } \\
\text { controle de pico de pressão inspiratória, } \\
\text { útil para compensar escape de ar em tubos } \\
\text { sem cuff e diminuir insuflação gástrica } \\
\text { associada a máscaras laríngeas e outros } \\
\text { dispositivos supraglóticos. Usualmente } \\
\text { utilizada em ventilação monopulmonar }\end{array}$ \\
\hline $\begin{array}{l}\text { VCV/V-CMV com Pressão garantida, } \\
\text { Autoflow ou Regulada a pressão, com } \\
\text { volume controle }\end{array}$ & Mandatória & $\begin{array}{l}\text { Ventilação controlada, limitada a } \\
\text { volume, ciclagem a tempo, pressão } \\
\text { limitada. Esse modo tem nomes } \\
\text { diferentes e algoritmos próprios em } \\
\text { diferentes fabricantes de ventiladores. } \\
\text { Propõe entregar o volume desejado } \\
\text { com a menor pressão inspiratória } \\
\text { possível }\end{array}$ & $\begin{array}{l}\text { Disponibilidade em ascensão nas } \\
\text { máquinas de ventilação, combina as } \\
\text { vantagens de VCV e PCV, permitindo um } \\
\text { controle acurado do volume corrente com } \\
\text { melhor comprometimento da pressão de } \\
\text { pico inspiratório }\end{array}$ \\
\hline
\end{tabular}

Continua. 
Conclusão.

Quadro 02. Principais modos ventilatórios usados em centro cirúrgico.

\begin{tabular}{|l|l|l|l|}
\hline Modo & Tipo & Detalhes & Uso em anestesia \\
\hline $\begin{array}{l}\text { V ou P-ACV (volume ou pressão } \\
\text { assistido-controlado) }\end{array}$ & $\begin{array}{l}\text { Assistido/ } \\
\text { Controlado }\end{array}$ & $\begin{array}{l}\text { Entrega o volume ou pressão } \\
\text { desejado, limitada tanto pelo esforço } \\
\text { inspiratório do paciente como } \\
\text { automaticamente }\end{array}$ & $\begin{array}{l}\text { Pode ser usado em pacientes com drive } \\
\text { respiratório residual para auxiliar na } \\
\text { respiração espontânea sem risco de apneia }\end{array}$ \\
\hline $\begin{array}{l}\text { V ou P-SIMV (volume ou pressão } \\
\text { ventilação sincronizada intermitente } \\
\text { mandatória) }\end{array}$ & $\begin{array}{l}\text { Assistido/ } \\
\text { Controlado }\end{array}$ & $\begin{array}{l}\text { Entrega o volume ou pressão } \\
\text { desejados mandatoriamente, mais um } \\
\text { volume ou pressão assistido quando } \\
\text { se detecta um esforço inspiratório } \\
\text { dentro de uma janela de sensibilidade }\end{array}$ & $\begin{array}{l}\text { Similar ao V/P-AVC, pode ser usado em } \\
\text { pacientes com drive respiratório residual, } \\
\text { ou ao despertar de anestesia geral }\end{array}$ \\
\hline $\begin{array}{l}\text { PSV (ventilação de pressão de } \\
\text { suporte) }\end{array}$ & Assistido & $\begin{array}{l}\text { Entrega a pressão desejada quando } \\
\text { detecta esforço inspiratório }\end{array}$ & $\begin{array}{l}\text { Em pacientes com drive respiratório, na } \\
\text { presençadedoençarestritivapulmonar, indução } \\
\text { de anestesia geral, doença neuromuscular, } \\
\text { desmame de ventilação controlada }\end{array}$ \\
\hline CPAP & Assistido & $\begin{array}{l}\text { Aumenta a pressão de entrada em } \\
\text { via aérea de pacientes respirando } \\
\text { espontaneamente }\end{array}$ & $\begin{array}{l}\text { Na indução, para evitar perda excessiva } \\
\text { de capacidade residual funcional, pode } \\
\text { diminuir o esforço inspiratório em } \\
\text { pacientes intubados antes da extubação }\end{array}$ \\
\hline
\end{tabular}

Tabela adaptada de: Ball L, Dameri M, Pelosi P. Modes of mechanical ventilation for the operating room. Best Practice \& Research Clinical Anaesthesiology. 2015;29:285-289

\section{Ventilação mecânica e comorbidades}

Um número significativo de pacientes submetidos a ventilação mecânica para cirurgia sofrem de alguma comorbidade, várias destas foram identificadas como associadas a um aumento do risco de CPPs, como tabagismo, ${ }^{12,14-17} \mathrm{DPOC},{ }^{12,14-17}$ hipertensão pulmonar, ${ }^{12,14}$ SDRA, $^{14}$ AOS, ${ }^{12,14-17}$ doença cardíaca $^{13,14}$ e neurológica. ${ }^{13,14}$ Entretanto, evidências da literatura para os benefícios das EVP em pacientes com comorbidades específicas são limitados. ${ }^{13}$

Doentes com DPOC apresentam inflamação crônica das vias aéreas e parênquima pulmonar, resultando em aumento da resistência das vias aéreas e diminuição da força elástica pulmonar. ${ }^{12,13,16}$ Isso leva ao colapso das vias aéreas no final da expiração resultando em uma expiração incompleta, maior volume residual expiratório final, hiperinflação e auto-PEEP. Por sua vez, pode gerar hipoxemia e hipercapnia. ${ }^{13}$ Pacientes com DPOC devem estar em condição clínica estável para se submeter a uma cirurgia eletiva. Em caso de exacerbações, a cirurgia deve ser adiada até compensação do quadro clínico. No entanto, mesmo pacientes estáveis tem o risco até quatro vezes maior de desenvolver CPPs. ${ }^{12,13,16}$ Esse risco parece ser menor, se o volume expiratório forçado no primeiro segundo $\left(\mathrm{VEF}_{1}\right)$ é maior que $60 \% .{ }^{13}$ Nas exacerbações agudas do DPOC com necessidade de suporte ventilatório, o uso de ventilação não invasiva (VNI) reduz a mortalidade..$^{13,19}$

Evidências também sugerem que evitar anestesia geral e intubação resultam em menor incidência de pneumonia, falência do desmame ventilatório e intubação não planejada no pós-operatório em pacientes com DPOC. ${ }^{12,13}$ Quando se faz necessário a anestesia geral para cirurgias abdominais de grande porte, é recomendado associação com peridural, ${ }^{12,13,16}$ pois reduz a mortalidade a quase metade em 30 dias. ${ }^{13}$ Quanto mais grave o DPOC, mais os pacientes parecem se beneficiar da anestesia peridural associada. ${ }^{13}$
A aplicação de PEEP pode causar superdistensão e auto-PEEP em pacientes com DPOC e precisa ser titulada individualmente nesses pacientes para não causar barotrauma. Além disso, parece haver uma melhor distribuição do fluxo inspiratório de ar com o modo de PCV que outros modos. ${ }^{13}$

Inflamação crônica das vias aéreas também ocorre em pacientes com asma, que apresentam uma maior reatividade brônquica e maior risco de desenvolverem broncoespasmo durante anestesia. ${ }^{12,13,16} \mathrm{O}$ broncoespasmo tem tratamento específico e imediato, não acarretando risco adicional no pósoperatório. Portanto, em adultos com asma controlada, o risco de CPPs não é maior do que a população geral. ${ }^{13,14}$

Uma pressão arterial pulmonar média maior que $25 \mathrm{mmHg}$ configura hipertensão pulmonar (HP), o que aumenta o risco de morbimortalidade em até 15 vezes. ${ }^{13}$ Pacientes com HP devem ser vistos por um especialista antes da cirurgia para que se apresentem na melhor condição clínica possível para cirurgia. ${ }^{12,13}$ Deve-se evitar no intraoperatório hipoxemia, hipercapnia e acidose, pois aumentam ainda mais a hipertensão pulmonar. Geralmente, a normocapnia pode facilmente ser atingida com $\mathrm{VC}$ de 7 e $8 \mathrm{ml} / \mathrm{kg}$ de peso ideal. O uso de PEEP e manobras de recrutamento alveolar devem ser realizados com extrema cautela, pois podem causar aumento de pós-carga de ventrículo direito. ${ }^{13}$

Pacientes com apneia obstrutiva do sono tem maior risco de CPPs. ${ }^{12,13,16}$ Aincidência deAOS pode variarde $2 \%$ a $25 \%$, podendo chegar a $70 \%$ em obesos mórbidos. Quanto mais episódios de hipopneia/apneia, maior o risco de complicações gerais pósoperatórias. ${ }^{13}$ As diretrizes atuais também recomendam o uso de técnicas neuroaxiais quando possível. O uso de Continuous Positive Airway Pressure (CPAP) é altamente recomendado, principalmente se já em uso prévio pelo paciente. . $^{12,13-15,19}$

Pacientes com doença cardíaca são tipicamente considerados ASA $>2$, o que por sua vez já está associado com risco de CPPs. 
Manobras de recrutamento devem ser realizadas com cautela e sob monitorização, pois podem desenvolver hipotensão sistêmica, necessidade de vasopressores e acidose metabólica. A acidose aumenta a susceptibilidade de arritmias, como fibrilação ventricular, e deixa o miocárdio menos sensível ao estimulo do marca-passo. ${ }^{13}$

Já as doenças neurológicas podem aumentar o risco de CPPs devido à fraqueza muscular e desregulação da respiração e deglutição. Em pacientes com aumento da pressão intracraniana, a ventilação intraoperatória com baixos volumes deve ser realizada sob monitorização cuidadosa da $\mathrm{PaCO}_{2}$ e da pressão intracraniana, especialmente durante as manobras de recrutamento. Até o momento, não existem dados disponíveis para evidenciar se baixo $\mathrm{VC}$ apresenta melhor resultado em pacientes neurocirúrgicos. ${ }^{13}$

Pacientes com SDRA se beneficiam quando ventilados com $\mathrm{VC}$ de $6 \mathrm{ml} / \mathrm{kg}$ de peso corporal ideal, pressões de platô entre 25 e $30 \mathrm{cmH}_{2} \mathrm{O}$, PEEP ajustada entre 5 e 25 dependendo do índice de oxigenação. A meta de oxigenação é alcançar valor de $\mathrm{PaO}_{2}$ entre 55 e $80 \mathrm{mmHg}$. Caso esses pacientes tenham que ser operados, o objetivo é manter as configurações do ventilador já em curso. Uma eventual desconexão do tubo traqueal pode resultar em deterioração da função pulmonar, portanto é aconselhável transportar o ventilador de cuidados intensivos para a sala de cirurgia. ${ }^{13}$

\section{Ventilação monopulmonar (VMP)}

Ventilação monupulmonar consiste na técnica de supressão da ventilação de um dos pulmões através do uso de bloqueadores endobrônquicos ou cateteres de intubação duplo lúmem. A ventilação é interrompida, enquanto a perfusão é mantida, causando um efeito shunt importante. Hipoxemia pode vir ocorrer e é causada não só pelo shunt intrapulmonar como também pelo colabamento alveolar do pulmão dependente..$^{23}$ $\mathrm{Na}$ tentativa de diminuir o shunt, ocorre a vasoconstrição pulmonar hipóxica que é um mecanismo que reduz o fluxo sanguíneo luxuriante para o pulmão não ventilado. ${ }^{11,23}$

Embora a incidência de hipoxemia intraoperatória durante a ventilação monopulmonar tenha diminuído de 20 a $25 \%$ em 1970 para cerca de $10 \%$ atualmente, ainda é um desafio para anestesiologistas. O deslocamento do dispositivo de intubação seletiva é muito comum, sendo também uma causa de hipoxemia (cerca de 38\%). Portanto é crucial checar a localização correta do tubo com broncoscopia de fibra óptica caso ocorra dessaturação. ${ }^{23}$

Historicamente, a abordagem clássica diante da ventilação monopulmonar era destinada principalmente a prevenção e tratamento da hipoxemia aguda. A recomendação clássica era usar uma $\mathrm{FiO}_{2}$ de $100 \%$ para dar uma maior margem de segurança e também causar vasodilatação no pulmão ventilado. ${ }^{14}$ Entretanto, mesmo por um curto período, altas frações de oxigênio causam atelectasia, e as manobras de recrutamento alveolar não são tão eficazes quando executadas com alta $\mathrm{FiO}_{2} \cdot{ }^{11,19,23}$ Portanto, é conveniente manter uma $\mathrm{FiO}_{2}$ o mais baixo possível e aumentar se necessário.
Um VC entre 6 e $8 \mathrm{ml} / \mathrm{kg}$ de peso ideal é considerado estratégia protetora para dois pulmões s,21,22 $^{50}$ se aplicar esse volume em apenas um pulmão, pode ser um valor duplamente elevado e não exercer mais seu efeito protetor. No entanto, ao se reduzir para metade o VC (3 a $4 \mathrm{ml} / \mathrm{kg}$ ), esse valor se aproximaria muito do volume do espaço morto. Empiricamente, um VC de 5 e $6 \mathrm{ml} / \mathrm{kg}$ parece ser conveniente, mas precisa de mais estudos para sua confirmação. ${ }^{23}$

A aplicação da PEEP no pulmão ventilado durante a abordagem tradicional era utilizada apenas caso o paciente apresentasse hipoxemia, e CPAP no pulmão não dependente, apenas se hipoxemia refrataria. Para evitar um desvio do fluxo sanguíneo para o pulmão não ventilado, o nível da PEEP não deve exceder a CPAP. Em alguns casos, o uso de PEEP pode levar a compressão dos vasos perialveolares, desvio do fluxo sanguíneo para o pulmão não ventilado e consequente diminuição de $\mathrm{PaO}_{2}$. No entanto, também foi evidenciado que o uso da PEEP durante a ventilação monopulmonar pode aumentar a CRF, melhorar a relação ventilação/perfusão e evitar o colapso alveolar ao final da expiração. ${ }^{23}$ Todavia, os efeitos de diferentes níveis de PEEP para proteção pulmonar durante VMP ainda exige uma investigação mais aprofundada do assunto.

Para reabertura de alvéolos atelectasiados a PEEP é ineficaz. Para isso, são necessárias manobras de recrutamento alveolar. Foi mostrado que MR melhoram a oxigenação e diminuem o espaço morto, resultando num aumento da eficácia da ventilação. ${ }^{23}$ Quanto ao uso de CPAP no pulmão não dependente, vários estudos demonstraram claramente que seu uso melhora a $\mathrm{PaO}_{2}$. Um CPAP de $5 \mathrm{cmH} 2 \mathrm{O}$ durante uma toracotomia aberta não interfere na exposição cirúrgica, porém, durante uma cirurgia vídeo-assistida o colapso pulmonar é necessário. ${ }^{23} \mathrm{Em}$ um estudo clínico aleatório randomizado, o uso de CPAP foi associado a uma menor resposta inflamatória local durante VMP para esofagectomia. ${ }^{25}$ Esse achado reforça a ideia que a aplicação de CPAP no pulmão não dependente impede o colapso alveolar completo, sendo benéfico tanto para uma melhor oxigenação quanto para proteção pulmonar. 18,19,21,22

Foi demostrado ainda que a aplicação de ventilação a jato de alta frequência (VJAF) tem efeito benéfico comparável ao uso de CPAP, melhorando o nível de oxigenação. Todavia, devido à simplicidade e familiaridade maior com o CPAP, o uso de VJAF ficou limitado apenas quando aquela não pode ser mantida, como em pacientes com brônquios abertos cirurgicamente. $^{23}$

Curiosamente, foi constatado através de exames de imagens pulmonares no pós-operatório que o pulmão ventilado apresentava mais injúria que o pulmão operado. Isso reforça a importância de se adotar EVP em cirurgias torácicas. ${ }^{23}$ Algumas estratégias não ventilatórias podem também ser enquadradas em medidas protetoras contra injúria pulmonar, como gerenciamento de fluido ideal guiado por metas, ${ }^{12,16,23}$ proteção da camada de glicocálice ${ }^{14}$ e realização de anestesia peridural. ${ }^{16,23}$

\section{Ventilação mecânica intraoperatória no paciente pediátrico}

É preciso estar ciente das diferenças entre o sistema respiratório 
de crianças e adultos ao se aplicar VM na faixa pediátrica. Durante a primeira infância, o desenvolvimento respiratório se dá de forma heterogênea, com maior crescimento do pulmão distal em relação às vias aéreas. Com esse desenvolvimento diferencial, há um aumento da complacência pulmonar e redução da resistência das vias aéreas rapidamente com o aumento da altura da criança. Como consequência, há um aumento da CRF e volume residual (VR); e diminuição da capacidade de fechamento (CF) pulmonar com o aumento da idade da criança. ${ }^{22}$

A parede torácica na primeira infância impõe menor oposição ao colapso pulmonar, resultando em baixo volume pulmonar. Durante a faixa pré-escolar, devido à ossificação das costelas e aumento da massa muscular, a parede torácica se torna mais rígida, atuando de forma contraria à força de recuo elástica pulmonar e impedindo o colabamento dos alvéolos. ${ }^{22}$

Dados clínicos são escassos quanto ao acometimento de LPIV no contexto pediátrico. Dessa forma, a prática adotada em adultos acaba sendo extrapolada para criança. Recentemente, uma revisão sistemática analisou 1756 pacientes pediátricos ventilados no intraoperatório com VC de 7, 8,10 e $12 \mathrm{ml} / \mathrm{kg}$. Não foi encontrada nenhuma associação entre mortalidade e o VC utilizado. Assim, pode-se perceber que a susceptibilidade para LPIV é menor na faixa etária pediátrica. Curiosamente, estudos realizados com ratos e camundongos também evidenciaram uma maior injuria em pulmões adultos. ${ }^{22}$

No momento, dada a falta de evidencias científicas, não podem ser feitas recomendações sobre VC ideal na ventilação pediátrica. Pode-se considerar que um VC entre 6 e $10 \mathrm{ml} /$ $\mathrm{kg}$ seja aceitável, mas VC maiores que $10 \mathrm{ml} / \mathrm{kg}$ devem ser evitados. ${ }^{22}$ Para a prevenção de atelectrauma a aplicação de PEEP parece racional, porém, o nível ótimo de PEEP também não está claro na população infantil. Foi relatado em estudo recente uma diminuição de $10 \%$ do debito cardíaco com uso de PEEP em 55 crianças. O estudo sugere aos profissionais da saúde a não utilização de PEEP. No entanto, como regra geral, a PEEP deve ser individualizada, procurando encontrar um equilíbrio ótimo entre a hemodinâmica e a oxigenação. Isso indica, que uma PEEP entre 4 e $8 \mathrm{cmH} 2 \mathrm{O}$ podem ser usados. Níveis mais altos podem ser necessários em pacientes com lesão pulmonar ${ }^{22}$ e obesos. ${ }^{19}$

Quanto ao modo ventilatório, pode ser recomendado a ventilação controlada a pressão (PVC) devido o padrão de fluxo em desaceleração. O modo $\mathrm{PVC}$ requer que o médico determine a pressão inspiratória em vez do VC. O VC administrado vai variar conforme a complacência e resistência das vias aéreas. Dessa forma, o VC deve ser vigiado de perto para mantê-lo na faixa desejada. ${ }^{19,22}$

Infelizmente, a VM em pediatria é pouco apoiada em evidência. Isso significa que há necessidade de estudos clínicos para se compreender melhor a VM em pacientes pediátricos.

\section{Estratégias ventilatórias em cirurgia cardíaca}

Apesar dos avanços tecnológicos, a cirurgia cardíaca com ou sem circulação extracorpórea (CEC) ainda possui morbidade respiratória relevante, levando a cuidados intensivos prolongados e maior permanência hospitalar. A morbidade pulmonar pode se manifestar desde atelectasias à SDRA..$^{5,14,21}$ Durante a CEC, há o hábito de desconexão do ventilador ao paciente, ficando o pulmão susceptível ao colapso. Estudos mostram que manter a ventilação com baixos volumes $(5 \mathrm{ml} / \mathrm{kg})$ durante esse período apresentou melhor oxigenação e redução da liberação de mediadores inflamatórios. A CEC também gera redução do fluxo sanguíneo pulmonar, podendo ocorrer lesões isquêmicas, e posterior fenômeno de isquemia e reperfusão. Isso contribui ainda mais para inflamação local e sistêmica, culminando com complicação pulmonar pós-operatória. ${ }^{21}$ Após cirurgia cardíaca, o uso de $\mathrm{VC}$ elevados $(>12 \mathrm{ml} / \mathrm{kg})$ foi associado a falência de órgãos, VM prolongada e permanência longa em UTI. ${ }^{5,21}$ Em um estudo randomizado 149 pacientes de cirurgia cardíaca eletiva foram divididos em dois grupos. Um foi ventilado com $6 \mathrm{ml} / \mathrm{kg}$ e outro com $10 \mathrm{ml} / \mathrm{kg}$. O nível de PEEP foi igual em ambos. Os pacientes que receberam VC mais baixos tiveram maior probabilidade de extubação em 6 horas após cirurgia e também apresentaram uma menor taxa de reintubação. ${ }^{21}$

Para se garantir a manutenção da ventilação alveolar e excreção de dióxido de carbono $\left(\mathrm{CO}_{2}\right)$, pode haver necessidade de se aumentar ligeiramente a frequência respiratória. Geralmente, frequências menores que 20 incursões por minuto não culminam em auto-PEEP e mantém o $\mathrm{CO}_{2}$ dentro da faixa adequada. $^{21}$

Poucos estudos avaliaram o impacto da PEEP e MR em pacientes de cirurgia cardíaca. Autores constataram aumento da mortalidade quando baixo PEEP é associada a baixo VC. Portanto, valores de PEEP levemente acima de $5 \mathrm{cmH} 2 \mathrm{O}$ provavelmente seja o recomendado. Quanto a MR, deve-se ter cautela, pois alterações hemodinâmicas podem vir a ocorrer em um sistema cardiovascular já comprometido. Contudo, as MR podem vir a ser necessárias para reabrir áreas do pulmão não ventiladas ou pouco ventiladas durante cirurgias cardíacas, ou após a desconexão com o ventilador após chegada a UTI. ${ }^{21}$

Quanto a $\mathrm{FiO}_{2}$, valores acima de $60 \%$ estão relacionados a atelectasia de reabsorção, mas valores acima de $80 \%$ apresentam o efeito mais significativo. ${ }^{1}$ Além disso, não há benefício manter $\mathrm{PaO}_{2}$ do paciente acima de $100 \mathrm{mmHg}$, pois a hiperóxia pode causar vasoespasmo das artérias coronárias e carótida. Geralmente, a recomendação é titular a $\mathrm{FiO}_{2}$ com o objetivo de manter a $\mathrm{SpO}_{2}$ maior que $92 \%$. $^{21}$

\section{CONCLUSÃO}

A melhor compreensão dos mecanismos de lesão pulmonar conduziu a realização de uma série de estudos nas últimas décadas. Os resultados dessas pesquisas têm atualizado o modo de como é realizada a VM no intraoperatório. Redução das complicações pós-operatórias devido a melhores estratégias ventilatórias podem afetar um grande número de pacientes, reduzir os custos de cuidados de saúde e contribuir para um melhor resultado clínico. No entanto, estudos mais aprofundados precisam ser realizados para definir o nível de PEEP ideal e o real benefício das manobras de 
recrutamento alveolar. Também são necessários mais estudos que comprovem os benefícios das estratégias de ventilação protetora nas subespecialidades.

\section{REFERÊNCIAS}

1. Serpa A Neto, Hemmes SN, Abreu MG, Pelosi P, Schultz MJ; PROVE Network investigators. Protocol for a systematic review and individual patient data meta-analysis of benefit of so-called lungprotective ventilation settings in patients under general anesthesia for surgery. Syst Rev. 2014;3:2.

2. Tao T, Bo L, Chen F, Xie Q, Zou Y, Hu B, et al. Effect of protective ventilation on postoperative pulmonary complications in patients undergoing general anaesthesia: a meta-analysis of randomised controlled trials. BMJ Open. 2014;4(6):1-8.

3. Serpa A Neto, Schultz MJ, Slutsky AS. Current concepts of protective ventilation during general anaesthesia. Swiss Med Wkly. 2015;145:1-7.

4. Futier E, Constantin JM, Jaber S. Protective lung ventilation in operating room: a systematic review. Minerva Anestesiol. 2014;80(6):726-35.

5. Serpa A Neto, Schultz MJ, Gama de Abreu M. Intraoperative ventilation strategies to prevent postoperative pulmonary complications: systematic review, meta-analysis, and trial sequential analysis. Best Pract Res Clin Anaesthesiol. 2015;29(3):331-40.

6. Hemmes SN, Serpa A Neto, Schultz MJ. Intraoperative ventilatory strategies to prevent postoperative pulmonary complications: a metaanalysis. Curr Opin Anaesthesiol. 2013;26(2):126-33.

7. Severgnini P, Selmo G, Lanza C, Chiesa A, Frigerio A, Bacuzzi A, et al. Protective mechanical ventilation during general anesthesia for open abdominal surgery improves postoperative pulmonary function. Anesthesiology. 2013;118(6):1307-21.

8. Futier E, Constantin JM, Paugam-Burtz C, Pascal J, Eurin M, Neuschwander A, et al. A trial of intraoperative low-tidal-volume ventilation in abdominal surgery. N Engl J Med. 2013;369(5):428-37.

9. Ge Y, Yuan L, Jiang X, Wang X, Xu R, Ma W. [Effect of lung protection mechanical ventilation on respiratory function in the elderly undergoing spinal fusion]. Journal of Central South University. 2013;38(1):81-5. Chinese.

10. Güldner A, Kiss T, Serpa A Neto, Hemmes SN, Canet J, Spieth PM, et al. Intraoperative protective mechanical ventilation for prevention of postoperative pulmonary complications: a comprehensive review of the role of tidal volume, positive end-expiratory pressure, and lung recruitment maneuvers. Anesthesiology. 2015;123(3):692-713.

11. Hedenstierna G, Edmark L. Effects of anesthesia on the respiratory system. Best Pract Res Clin Anaesthesiol. 2015;29(3):273-84.

12. Taylor A, DeBoard Z, Gauvin JM. Prevention of postoperative pulmonary complications. Surg Clin North Am. 2015;95(2):237-54.

13. Treschman TA, Malbouisson LM, Beiderlinden M. Intraoperative mechanical ventilation strategies to prevent postoperative pulmonary
Os conhecimentos recentes sobre este tema devem levar nos próximos anos a maiores alterações na nossa prática diária.

complications in patients with pulmonary and extrapulmonary comorbidities. Best Pract Res Clin Anaesthesiol. 2015;29(3):341-55.

14. Gallart L, Canet J. Post-operative pulmonary complications: understanding definitions and risk assessment. Best Pract Res Clin Anaesthesiol. 2015;29(3):315-30.

15. Qaseem A, Snow V, Fitterman N, Hornbake ER, Lawrence VA, Smetana GW, et al. Risk assessment for and strategies to reduce perioperative pulmonary complications for patients undergoing non cardiothoracic surgery: a guideline from the American College of Physicians. Ann Intern Med. 2006;144(8):575-80.

16. Güldner A, Spieth PM, Abreu MG. Non-ventilatory approaches to prevent postoperative pulmonary complications. Best Pract Res Clin Anaesthesiol. 2015;29(3):397-410.

17. Mazo V, Sabate S, Canet J, Gallart L, Abreu MG, Belda J, et al. Prospective external validation of a predictive score for postoperative pulmonary complications. Anesthesiology. 2014;121(2):219-31.

18. Silva PL, Negrini D, Rocco P. Mechanisms of ventilator-induced lung injury in healthy lungs. Best Pract Res Clin Anaesthesiol. 2015;29(3):301-13.

19. Ball L, Dameri M, Pelosi P. Modes of mechanical ventilation for the operating room. Best Pract Res Clin Anaesthesiol. 2015;29(3):285-99.

20. Bendixen HH, Hedley-Whyte J, Laver MB. Impaired oxygenation in surgical patients during general anesthesia with controlled ventilation. A concept of atelectasis. N Engl J Med. 1963;269(19):991-6.

21. Lellouche F, Delorme M, Bussières J, Ouattara A. Perioperative ventilatory strategies in cardiac surgery. Best Pract Res Clin Anaesthesiol. 2015;29(3):381-95.

22. Kneyber MC. Intraoperative mechanical ventilation for the pediatric patient. Best Pract Res Clin Anaesthesiol. 2015;29(3):371-9.

23. Sentürk M, Slinger $P$, Cohen E. Intraoperative mechanical ventilation strategies for one-lung ventilation. Best Pract Res Clin Anaesthesiol. 2015;29(3):357-69.

24. PROVE Network Investigators for the Clinical Trial Network of the European Society of Anaesthesiology; Hemmes SN, Abreu MG, Pelosi P, Schultz MJ. High versus low positive end-expiratory pressure during general anaesthesia for open abdominal surgery (PROVHILO trial): a multicentre randomised controlled trial. Lancet. 2014;384(9942):495-503.

25. Verhage RJ, Boone J, Rijkers GT, Cromheecke GJ, Kroese $\mathrm{AC}$, Weijs TJ, et al. Reduced local immune response with continuous positive airway pressure during one-lung ventilation for oesophagectomy. Br J Anaesth. 2014;112(5):920-8.

\section{Como citar:}

Castro IN, Oliveira LA, Maia FL, Melo JR, Cavalcante FP. Papel atual das estratégias ventilatórias protetoras no período perioperatorio: artigo de revisão. Rev Med UFC. 2018 jan-mar;58(1):53-61. 